\title{
Efectos del consumo de marihuana en escolares sobre funciones cerebrales demostrados mediante pruebas neuropsicológicas e imágenes de neuro-SPECT
}

\author{
Ismael Mena, ${ }^{1,2}$ Anneliese Dörr, ${ }^{3,4}$ Sandra Viani, ${ }^{3,4}$ Sonia Neubauer, ${ }^{1}$ María Elena Gorostegui, ${ }^{3}$ \\ María Paz Dörr, ${ }^{3}$ Diana Ulloa ${ }^{3}$
}

Artículo original

\section{SUMMARY}

Comparative study based on 565 adolescent school children coming from four schools in the metropolitan area of Santiago, Chile. All were interviewed in order to select a sample that was stratified for sex, class and condition of consumer or non-consumer. The variables of intellectual coefficient and socioeconomical level were maintained constant. With this selection we conformed two groups: 40 consumers exclusively of marihuana and 40 non-consumers. We compared the results obtained in both groups in the neuropsychological tests while the neuroSPECT studies of consumers were compared against a normal database for the same age group. The adolescent consumers of marihuana demonstrate less cognitive capacity related to the process of learning such as attention, concentration, ranking, viso-spatial integration, immediate retention and visual memory. The differences between both groups are statistically significant.

The findings of neuroSPECT demonstrate subgenual hypoperfusion bilaterally, more marked on the left side, in area 25 of Brodmann. This area controls mood. There is also frontal bilateral hypoperfusion (area 10 and area 32 of Brodmann). Area 32 is pre-anterior cingulate gyrus. Also hypoperfusion of the anterior cingulate gyrus (area 24 of Brodmann) and hypoperfusion of area 36 of Brodmann that projects over the hippocampus.

Students that were consumers exclusively of marihuana demonstrate coincident abnormal findings of neuroimages and neuropsychological tests in areas of the brain related with learning and also significant differences between consumers with non-consumers in the neuropsychological tests.

Key words: Cannabis, marihuana, adolescent, SPECT, HMPAO, neuropsychological tests.

\section{RESUMEN}

Estudio comparativo basado en 565 escolares adolescentes pertenecientes a cuatro colegios de Santiago, Chile. Fueron encuestados todos para seleccionar una muestra estratificada por sexo, curso y condición de consumidores o no consumidores, manteniendo constante las variables coeficiente intelectual y nivel socioeconómico. Se conforman dos grupos: 40 consumidores exclusivos de marihuana y 40 no consumidores. Se comparan los resultados obtenidos en ambos grupos en los Test Neuropsicológios y del NeuroSPECT de consumidores con una base de datos considerados normales para el mismo grupo etario.

Los adolescentes consumidores de marihuana evidencian menores habilidades cognitivas asociadas al proceso de aprendizaje, tales como atención, concentración, jerarquización, integración visoespacial, retención inmediata y memoria visual. Las diferencias entre ambos grupos son estadísticamente significativas.

Los hallazgos del NeuroSPECT muestran hipoperfusión subgenual bilateral, más marcada en el hemisferio izquierdo (área 25 de Brodmann), hipoperfusión frontal bilateral (areas 10 y 32 de Brodmann), hipoperfusión del gyrus cingulado anterior (área 24 de Brodmann) e hipoperfusión del área 36 de Brodmann que proyecta sobre el hipocampo.

Los estudiantes consumidores exclusivamente de marihuana muestran compromiso coincidente en neuroimágenes y test neuropsicológicos en áreas del cerebro relacionadas con el aprendizaje y se diferencian significativamente de los no-consumidores en las pruebas neuropsicológicas.

Palabras claves: Cannabis, marihuana, adolescentes, NeuroS$\mathrm{PECT}, \mathrm{HMPAO}$, pruebas neuropsicológicas.

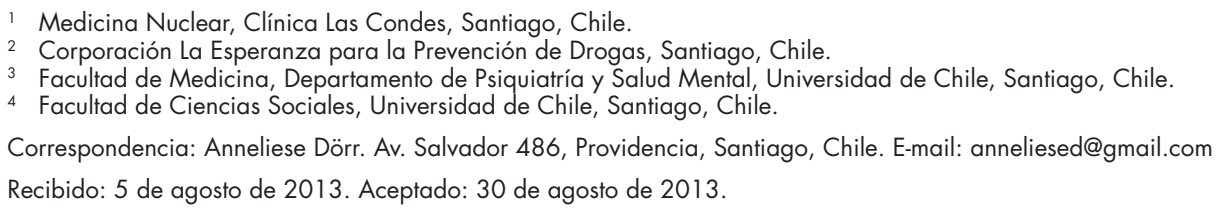




\section{INTRODUCCIÓN}

La impresión social en Chile acerca de la inocuidad del consumo de marihuana nos motivó a investigar su efecto sobre las funciones cognitivas necesarias para el aprendizaje escolar, sobre todo por las preocupantes estadísticas que muestran a este país como el de mayor consumo de marihuana en escolares en América Latina, así como una sostenida tendencia a disminuir la edad de inicio del consumo: se evidencia un $15.6 \%$ de consumidores entre los 13 a 18 años de acuerdo con el Informe Mundial sobre Drogas de 2010. ${ }^{1}$ El estudio Nacional de Drogas en Población Escolar concluyó un aumento de 4.4 puntos porcentuales en los últimos dos años del consumo experimental de marihuana entre los escolares de $8^{\circ}$ básico a $4^{\circ}$ medio, pasando de $15.6 \%$ en 2009 a $19.1 \%$ en 2011. El mismo informe dice que la falta de percepción de riesgo entre los estudiantes sobre el consumo frecuente de marihuana (una o dos veces a la semana), aumentó 10.2 puntos porcentuales, es decir, de $37.8 \%$ pasó a $48 \% .^{2}$ Estos datos son coincidentes y vienen a corroborar los hallazgos de investigaciones recientes, tales como la del estudio sobre marihuana y trastornos de aprendizaje realizado en 2007, en el que la percepción de peligro asociada al consumo era bajísima: en el grupo consumidor de marihuana sólo el 7\% desaprueba su consumo habitual, y en contraste el 74\% desaprueba el consumo de cigarrillos. ${ }^{3,4} \mathrm{Si}$ consideramos que la educación es el medio más efectivo para salir del círculo de la pobreza y que el mayor consumo ocurre en el nivel socioeconómico bajo, el hecho de consumir sin percepción de riesgo se torna más preocupante, quedando el joven consumidor expuesto a una situación de una mayor vulnerabilidad.

El Informe Mundial sobre Drogas de 2010 señala que la cannabis continúa siendo la droga que más se produce a nivel mundial y la sustancia ilícita de mayor consumo en casi todos los países del mundo. En la actualidad, entre 130 y 190 millones de personas la fuman al menos una vez por año. Aunque este nivel de consumo puede ser considerado bajo, es importante recordar que el riesgo de adicción depende no sólo de la cantidad, sino también de factores genético-sociales y de la edad de inicio del consumo, además de la susceptibilidad personal. ${ }^{5}$ Asimismo, las tendencias globales muestran que conforme mejora el nivel de desarrollo económico de los países, aumenta el consumo de marihuana. ${ }^{1,6}$

La posibilidad de la despenalización del consumo ha puesto el tema de la marihuana y de otras drogas ilícitas en el centro de la atención de los medios. Sin embargo, en el debate faltan las consideraciones acerca de los efectos de la marihuana en los segmentos más vulnerables de la población, tales como los adolescentes escolares, que la consumen sin que sean rotulados aún como consumidores habituales o adictos. Ellos, por no caer en la categoría de "adictos", no son considerados en las políticas de salud pública y, por lo tanto, no cuentan con apoyo social, escolar, familiar o médico.
Varios autores se han referido a las consecuencias negativas de la marihuana en el aprendizaje y el rendimiento escolar, destacando que tanto los efectos "agradables" o "desagradables", como el riesgo de adicción y daño dependen de la susceptibilidad individual., ${ }^{3,-10}$ Otros daños reportados son los que afectan funciones propias de la corteza prefrontal, como la capacidad de planificación, de trabajo con propósito y control e inhibición de respuestas. ${ }^{11-13}$

Un efecto adicional, en relación al desempeño escolar, es el síndrome amotivacional o disminución de la iniciativa personal. ${ }^{14}$ Este cuadro es patognomónico del adolescente consumidor. Se caracteriza por deterioro en la conducta, pérdida de energía y abulia con importante limitación de las actividades habituales, lo que tiene relación con su incapacidad para proyectarse y organizar de manera eficiente el tiempo en pos de un determinado objetivo. Se suma un estado de pasividad e indiferencia caracterizado por disfunción generalizada de las capacidades sociales. ${ }^{15} \mathrm{El}$ síndrome amotivacional tiene efectos importantes dentro de lo que pudiera clasificarse como factores afectivos en el desempeño escolar en su conjunto.

Mediante neuroimágenes se han demostrado anormalidades de las estructuras, de las funciones en situación de reposo o bajo estímulo, de receptores y neurotransmisores en consumidores de sustancias ilícitas, incluida la marihuana. ${ }^{11,16}$ En especial, con imágenes funcionales de NeuroSPECT se demostró el efecto neurotóxico irreversible de la cocaína con alteraciones multifocales del flujo sanguíneo cerebral, de distribución desorganizada y que se asocian con anormalidades funcionales cerebrales. ${ }^{17-19}$ Al utilizar en el análisis las áreas de Brodmann es posible correlacionar los hallazgos imagenológicos con el compromiso de las funciones correspondientes. ${ }^{20}$

La cannabis interviene indirectamente sobre la producción de dopamina e interactúa con receptores específicos CB1, los que se expresan intensamente en el hipocampo y en el cerebelo, lo que explica las implicancias de estas áreas en las alteraciones funcionales asociadas al consumo de esta droga. ${ }^{12,21}$

Estudios con técnicas de estimulación magnética transcraneana mostraron que cuando falla el sistema prefrontal, los sujetos comienzan a tomar decisiones destinadas a obtener gratificación inmediata, sin evaluación de las consecuencias. Esto correspondería a conductas guiadas preferentemente desde el sistema límbico. ${ }^{22,23}$ Los adolescentes, por la inmadurez de los lóbulos prefrontales propia de la edad, son más vulnerables a la hipofunción prefrontal causada por la marihuana y así a la determinación de su conducta por el sistema límbico, con las características antes señaladas. ${ }^{24}$

El Delta-9-tetrahidrocannabinol (THC) modifica la captación y el procesamiento de la información que realiza el hipocampo, crucial para el aprendizaje, la memoria, la integración de las experiencias sensoriales y de las motivaciones. ${ }^{25,26} \mathrm{El}$ THC es una molécula lipofílica que atraviesa 
con facilidad las barreras hematoencefálica y placentaria. Por esta afinidad a los lípidos se acumula en la grasa corporal, desde donde se libera paulatinamente provocando una prolongación de los efectos. Por eso, tras el consumo de un cigarrillo de marihuana, es posible detectar la presencia de metabolitos en la orina durante una semana. En consumidores crónicos, la orina puede ser positiva para THC hasta más de un mes después de suspender el consumo. ${ }^{5}$ El efecto de la droga sobre las funciones cognitivas persiste, en el consumidor, aun después de una abstinencia de varios días.

Nuestro objetivo fue evaluar los efectos sobre la función cerebral del consumo exclusivo de marihuana, excluyendo policonsumo, en adolescentes escolares no rotulados como adictos, mediante pruebas neuropsicológicas e imágenes cerebrales funcionales de NeuroSPECT.

\section{METODOLOGÍA}

\section{Selección de la muestra}

La muestra se seleccionó de una población conformada por los 565 alumnos de $1^{\circ}$ a $4^{\circ}$ año de enseñanza media de tres colegios públicos ubicados en comunas periféricas de Santiago, estratificada por sexo, curso y condición de consumo/no consumo de marihuana. Se mantienen constantes las variables: a) nivel socioeconómico medio bajo y b) nivel de desarrollo cognitivo, dentro de rangos de normalidad. ${ }^{27}$

Para efectos de este estudio se considera consumidor al alumno que declara un mínimo de cuatro episodios de consumo exclusivo de cannabis durante el último mes y consumo habitual mínimo de 18 meses. La muestra a evaluar en forma individual está compuesta por 40 sujetos consumidores exclusivamente de marihuana y 40 sujetos del grupo control sin antecedentes de consumo de ninguna droga. Sólo 29 de los 40 sujetos consumidores se estudiaron además con neuroSPECT. En ambos grupos la variable sexo fue distribuida proporcionalmente.

\section{Instrumentos y procedimientos}

Aplicación colectiva $(n=565)$

a) Consentimiento informado de la dirección de cada uno de los establecimientos participantes para la evaluación grupal de los 565 alumnos.

b) Cuestionario de Evaluación Psicosocial y Consumo de Dörr et al. abreviado y adaptado, destinado a recolección de datos demográficos, conductas de consumo y otras informaciones requeridas, para determinar la composición de la muestra. ${ }^{3}$

c) Cuestionario sobre antecedentes mórbidos del estudiante, de su familia y respecto al promedio final de notas en los últimos cuatro años.

d) Test de Dominó (D-48).
Aplicación individual de pruebas neuropsicológicas al grupo consumidor $(n=40)$ y al grupo control no consumidor $(n=40)$

a) Consentimiento informado/asentimiento informado de los alumnos y de sus padres para la evaluación neuropsicólogica individual y aplicación de neuroSPECT. Los procedimientos contaron con la aprobación formal del Comité de Bioética de la Facultad de Medicina de la Universidad de Chile y del Comité de Etica de la Clínica Las Condes.

b) Test de Retención Visual Revisado de Benton (Benton, 1965).

c) Memoria de palabras de Rey (Rey, 1959).

d) Test de la Figura Compleja de Rey (Rey, 1959).

e) Test de Wisconsin (WSCT, 2001). Sólo se aplicó a los consumidores.

\section{NeuroSPECT al grupo consumidor $(n=29)$}

El neuroSPECT se realizó en un día normal de clases. Como preparación se evitó, desde 24 horas antes, el consumo de té, café, chocolate y bebidas de cola. Se administró $740 \mathrm{MBq}$ de Tc99m HMPAO (Ceretec Amersham) por via endovenosa en reposo, en un ambiente con baja luz y escaso ruido. Una hora después se realizó la adquisición de las imágenes tomográficas en un equipo Siemens ECAM de dos cabezales con colimadores de Baja Energía Alta Resolución.

En el procesamiento de NeuroSPECT se usó la reconstrucción tridimensional por retroproyección con filtro de $\mathrm{Bu}$ tterworth 4.25. Mediante normalización del volumen, usando la técnica de Tallairach (software de Segami Corp, Maryland, USA), se compara cada individuo con una base de datos normal del mismo grupo etario. Los resultados se expresan en desviaciones standard respecto a los normales para cada una de las 14000 unidades de volumen (voxel) en que se dividió el cerebro. Además se aplica una matriz con las áreas de Brodmann para el análisis regional de estos datos. ${ }^{20}$

\section{Análisis de los datos}

a) De la evaluación grupal se obtiene la muestra a ser evaluada en forma individual.

b) Se comparan los resultados en las pruebas neuropsicológicas de sujetos consumidores y no consumidores aplicando modelos de análisis estadístico clásicos y pruebas no paramétricas de contraste estadístico, por tamaño muestral.

c) Se analiza la información de neuroSPECT para los valores de perfusión cortical y sub-cortical expresados en porcentaje del máximo de referencia, calculando máximo, mínimo, promedio y desviación estandar (DS) en cada Área de Brodmann. Con el propósito de identificar subregiones de perfusión alterada dentro del volumen delimitado por las diferentes áreas de Brodmann (ROI), se trabajó con los valores de máximos o mínimos por área. 


\section{RESULTADOS}

De los 565 alumnos evaluados, 368 (65.1\%) señala no haber consumido droga en ninguna de sus formas y 197 (34.9\%) reconoce haberla "probado". Las edades de los grupos que conforman la muestra fluctuaron entre 15 y 18 años, siendo el promedio de edad del grupo consumidor de 16 años (cuadro 1).

Los resultados obtenidos en las pruebas neuropiscológicas, aplicadas en forma individual a los consumidores y no-consumidores seleccionados, muestran diferencias significativas en cuatro de las cinco pruebas aplicadas. En todas es mejor el rendimiento del grupo control (no consumidor) en comparación al grupo con consumo de marihuana.
Cuadro 1. Resultados de pruebas neuropsicológicas para consumidores $(n=40)$ y grupo control de no consumidores $(n=40)$

\begin{tabular}{|c|c|c|c|c|c|}
\hline \multirow[b]{2}{*}{ Pruebas } & \multirow[b]{2}{*}{ Control } & \multirow[b]{2}{*}{ Experimental } & \multicolumn{3}{|c|}{$\begin{array}{c}\text { Prueba t para } \\
\text { diferencia de medias }\end{array}$} \\
\hline & & & $T$ & Tc a 0.05 & $p$ \\
\hline Dominó & 113.0 & 106.8 & 1.92 & 2.02 & N.S. \\
\hline Rey Mem. Palabras & 7.4 & 6.3 & 2.90 & 1.99 & $<0.05$ \\
\hline Benton retención & 8.9 & 7.9 & 3.94 & 1.99 & $<0.05$ \\
\hline Benton error & 1.7 & 3.8 & -5.37 & 1.99 & $<0.05$ \\
\hline Rey Figura Compleja & 25.4 & 17.3 & 6.76 & 1.99 & $<0.05$ \\
\hline
\end{tabular}

N.S.: no significativo.

Memoria de palabras de Rey: Aparece disminuido en 15\% el desempeño de alumnos consumidores en pruebas que evalúan memoria verbal inmediata $(p<0.05)$.

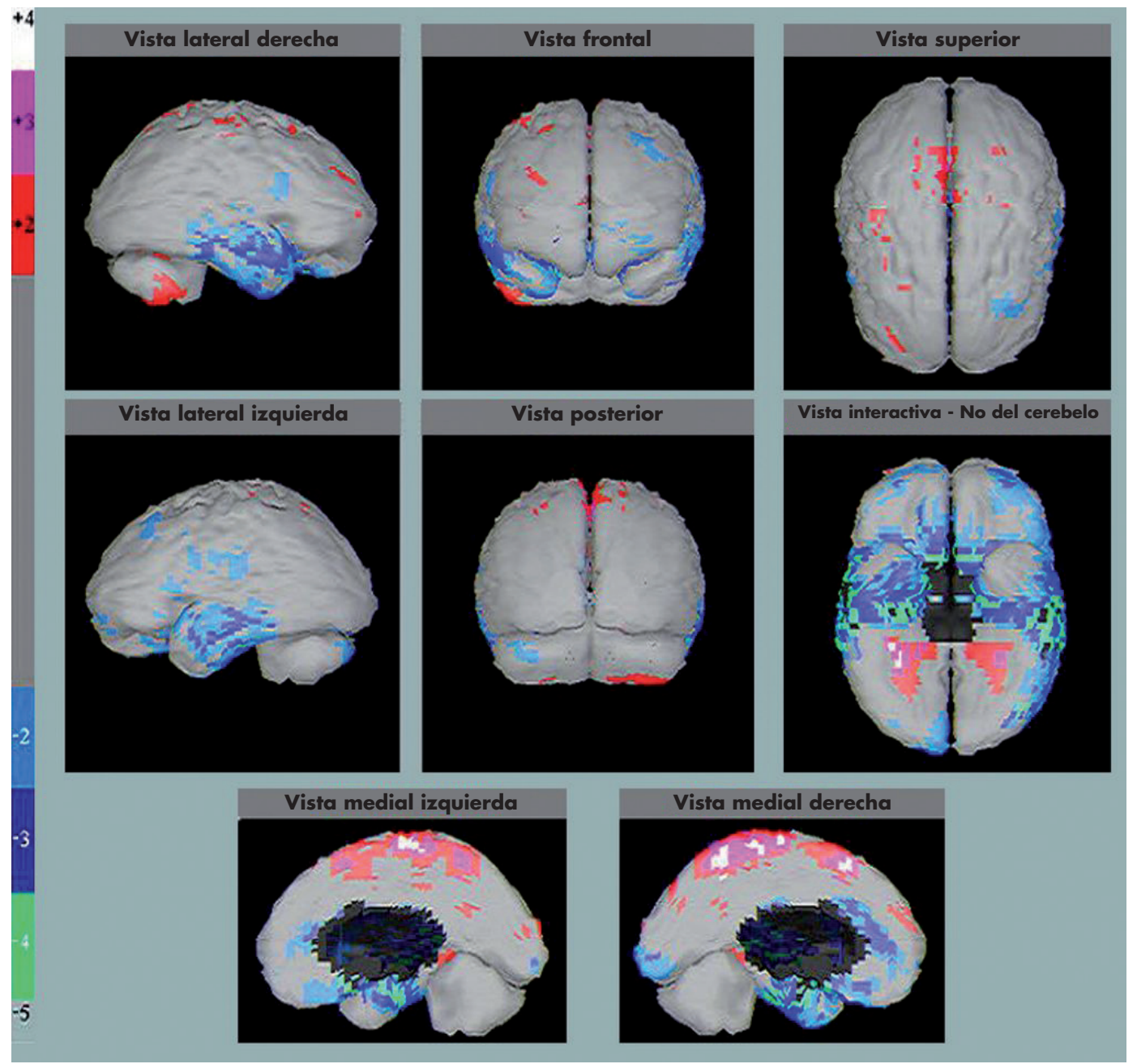

Figura 1. NeuroSPECT de escolar consumidor de marihuana exclusivamente. Se observan áreas de disminución de función a 2, 3 y 4 desviaciones estándar bajo el promedio normal (colores celeste, azul y verde) concentradas especialmente en ambos lóbulos temporales en el aspecto mesial y proyectando a ambos hipocampos. Se observa también hipoperfusión subgenual bilateral en el área 25 de Brodmann, que es un área que controla el ánimo. Se observa también hipoperfusión temporal lateral bilateral y áreas multifocales en la corteza frontal, además de compromiso de hipoperfusión en ambos cingulados anteriores. 
Cuadro 2. Prueba de Rey. Puntajes de error para grupo control $(n=40)$ y grupo experimental $(n=40)$

\begin{tabular}{|c|c|c|c|c|c|}
\hline \multirow{2}{*}{$\begin{array}{l}\text { Tipo } \\
\text { error }\end{array}$} & \multirow[b]{2}{*}{ Estrategia utilizada } & \multicolumn{2}{|c|}{ Control } & \multicolumn{2}{|c|}{ Experimental } \\
\hline & & $\mathrm{n}$ & $\%$ & $\mathrm{n}$ & $\%$ \\
\hline I & Construcción sobre rectángulo (adultos) & 16 & 40.0 & 2 & 5.0 \\
\hline ॥ & Comienza detalles con atención al rectángulo & 6 & 15.0 & 5 & 12.5 \\
\hline III & Contorno integral sin dif. Rectángulo & 2 & 5.0 & 3 & 7.5 \\
\hline IV & Detalles reconocibles sobre fondo confuso & 16 & 40.0 & 28 & 70.0 \\
\hline V & Yuxtaposición detalles ensayo-error (niños) & 0 & 0.0 & 2 & 5.0 \\
\hline VI & Asociación a esquema familiar. Recuerdo vago & 0 & 0.0 & 0 & 0.0 \\
\hline
\end{tabular}

Test de Retención Visual de Benton: Muestra puntajes significativamente superiores para los alumnos no consumidores en comparación a los consumidores en tareas que involucran capacidad de atención, concentración, retención inmediata, percepción, memoria visual y aptitudes visoconstructivas confirmando una alteración en los consumidores de la integración y estructuración de los estímulos espaciales. Los adolescentes consumidores cometen en promedio 3.8 errores por prueba contra 1.7 errores de los no consumidores. De esta manera, la cantidad de errores cometidos por el grupo consumidor resulta un $21 \%$ mayor. Esta diferencia es estadísticamente significativa $(\mathrm{p}<0.05)$, revelando un empobrecimiento de las capacidades de atención, concentra- ción y de la memoria espacial de corto alcance (cuadro 2).

Test de la Figura Compleja de Rey: Los puntajes obtenidos por ambos grupos muestran diferencias significativas a favor de los no-consumidores en tareas que involucran habilidad y estrategias de ejecución en el plano visoperceptivo, memoria visual, capacidad de jerarquización y organización de la información visual $(\mathrm{p}<0.05)$. Se encontró una diferencia cercana a 7 puntos en el promedio obtenido por ambos grupos, revelando claras dificultades de evocación y limitaciones en la fidelidad de la memoria visual de los consumidores. Los puntajes de la prueba identifican seis tipos de estrategia de ejecución de acuerdo a la edad para copia y memoria visual, desde las más elaboradas (Tipo I) a las más deficitarias (Tipo VI).

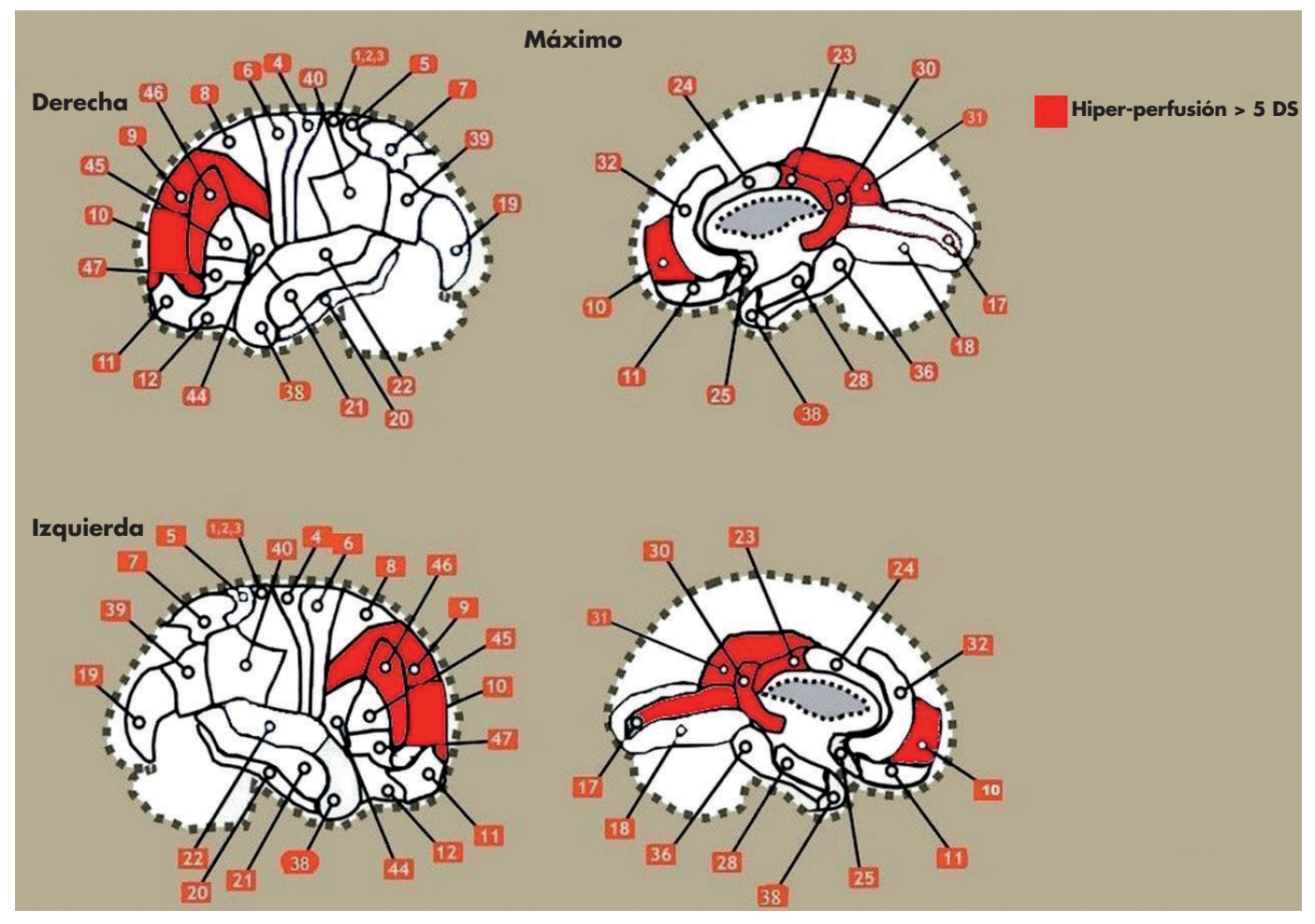

Figura 2. En 29 voluntarios expuestos a marihuana, se calcularon los máximos dentro de cada una de las áreas de Brodmann señaladas. Se consideró como anormal áreas que estaban a más de 5 desviaciones estándar sobre el promedio normal. Destacan en los lóbulos frontales el área 9,10 y 46 de Brodmann en la corteza ejecutiva, bilateralmente. También se observa aumento de perfusión en el cingulado posterior, área 30 de Brodmann, y en el área 31 , además de la corteza visual de asociación (área 17). 


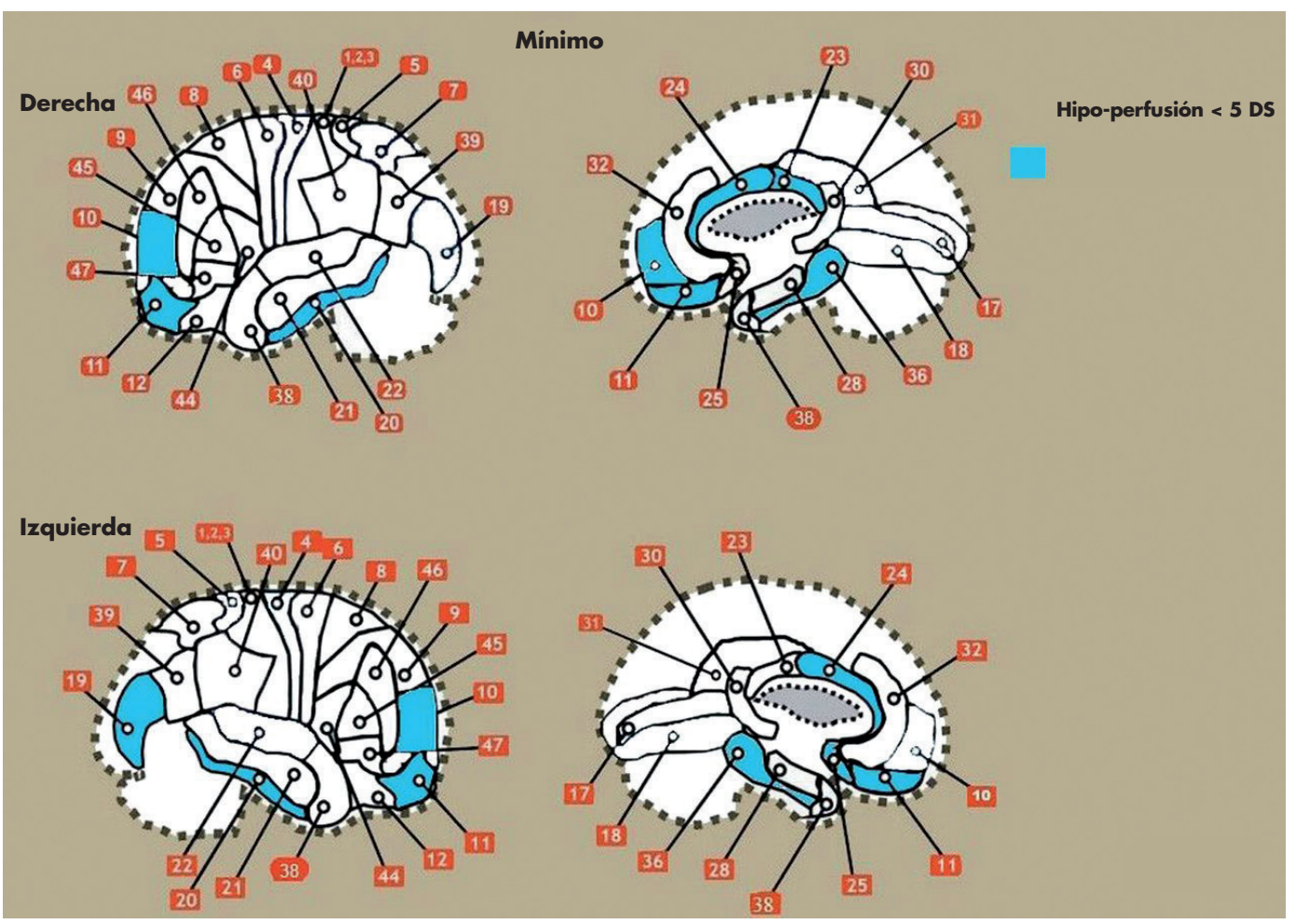

Figura 3. En 29 voluntarios expuestos a marihuana exclusivamente, se calcularon los mínimos dentro de cada una de las áreas de Brodmann señaladas y se destacan las áreas que estaban a menos de 5 desviaciones estándar bajo el promedio normal. Estas se encuentran en ambos lóbulos frontales, área 10 y 11 de Brodmann. También en el lóbulo temporal en los aspectos inferiores, área 20 de Brodmann, además de ambos cingulados anteriores, área 24 de Brodmann, y en el área 25 izquierda de Brodmann a nivel subgenual. Por último, se observa hipoperfusión en el área 36 de Brodmann bilateralmente que proyecta sobre el hipocampo.

Se entregan estos puntajes desagregados, por tipo de ejecución, para comparar el desempeño de ambos grupos. Del grupo control, 16 alumnos (40\%) alcanzan la estrategia constructiva tipo I, es decir, logran aplicar habilidades de jerarquización, elaboración perceptual y planificación. Del grupo experimental sólo dos alumnos $(5 \%)$ accede a este estilo de ejecución. Cabe destacar que el $70 \%$ del grupo consumidor desarrolla un estilo de ejecución Tipo IV, propia de una estrategia más concreta y ligada a inmadurez cognitiva.

El Test de Wisconsin mostró que, en la categoría errores totales, el 30\% del grupo consumidor se ubica en el nivel de deterioro moderado a intermedio. En errores perseverativos, el $26 \%$ está en nivel de deterioro medio o peor. Respecto al porcentaje de respuestas perseverativas, el $17.2 \%$ de los escolares consumidores obtienen puntuaciones de deterioro superior a la media. Estos resultados revelan, en casi un tercio del grupo consumidor, limitaciones en la capacidad de flexibilidad mental por alteraciones en las funciones ejecutivas que requieren estrategias de planificación, indagaciones organizadas y utilización del feedback ambiental para cambiar de esquema.

\section{Resultados de la evaluación con NeuroSPECT}

Se compararon los resultados individuales obtenidos con la población normal de la misma edad. Los resultados fueron expresados en Desviaciones Standard (DS) sobre y bajo el promedio normal (figura 1). Se proyectó una matriz de áreas de Brodmann sobre cada hemisferio y se calculó el promedio y la DS en 47 áreas, corticales y en cortes parasagitales. Se observó inicialmente que los defectos ocupaban parcialmente cada área por lo que se calcularon los valores mínimos y máximos del área de Brodmann (2.5\% mayor o menor del área respectivamente). Se consideraron como valores anormales los que eran mayores a 5 DS sobre o bajo el promedio normal. Las figuras 2 y 3 demuestran la heterogeneidad de las áreas afectadas con hipo o hiperperfusión. Se confirma que hay focos dentro de algunas áreas de Brodmann que están hiperperfundidas a 5 DS sobre el promedio normal: áreas 9, 10, 46 (lóbulo frontal) del hemisferio derecho, áreas 23,30 y 31 (cingulado posterior, circuito cognitivo) bilateralmente, y área 17 del hemisferio izquierdo, correspondiente al área visual de asociación (figura 2). 
Se observan focos de hipoperfusión, a menos de 5 DS bajo el promedio normal, bilateralmente en el área 24 de Brodmann, en el hemisferio izquierdo en área 25 de Brodmann, bilateralmente en la proyección del hipocampo y área 36 de Brodmann y en los lóbulos frontales en las áreas 10 y 11 de Brodmann. También se observa hipoperfusión profunda en el gyrus inferior temporal bilateral y 23 derecha (figura 3).

\section{CONCLUSIONES}

Se encuentra una asociación entre el consumo de marihuana en adolescentes y efectos nocivos sobre el funcionamiento cerebral, en especial en funciones cognitivas involucradas en el aprendizaje: memoria, atención, concentración y efectos negativos en cuanto a estilo de trabajo, precisión, organización del material, estrategias de ejecución y formas de abordaje de la tarea. La importancia, para los escolares, de la memoria verbal inmediata es clara: en la sala de clases la mayor parte de los conocimientos se entrega en forma oral. Al no contar con una adecuada memoria de trabajo, se dificulta el procesamiento de la información recibida por esta vía. A su vez, el número de errores que los jóvenes consumidores cometen en tareas que involucran atención y concentración, sumadas a deficiencias en las estrategias de trabajo, constituye un importante factor vinculado a sus problemas de rendimiento y fracaso escolar.

Dicha asociación se evidencia a través de las diferencias observadas en los resultados de las pruebas de evaluación neuropsicológica entre alumnos consumidores y no consumidores de marihuana. Los alumnos consumidores obtienen resultados comparativamente inferiores en todas las pruebas, con diferencias estadísticamente significativas. Esto permite establecer una clara asociación entre el consumo y la disminución significativa de puntajes obtenidos en las funciones cognitivas evaluadas, tanto en relación a lo esperado como a los resultados del grupo de pares no consumidores.

Mediante el NeuroSPECT se concluye que la marihuana produce, en la corte0za cerebral, alteraciones funcionales multifocales. Se compromete especialmente la cognición por hipoperfusión en la proyección del hipocampo (área 36 de Brodmann), el control del ánimo por compromiso del área 25 de Brodmann en el hemisferio izquierdo y la función ejecutiva con anormalidad frontal en área 10 y 11 de Brodmann bilateral. Hay que destacar que la corteza frontal participa en la gama de conductas humanas relacionadas con la dimensión ética, función que también incidiría en el trabajo y la conducta social de los escolares que consumen marihuana. ${ }^{9}$

Llama la atención que, a diferencia de estudios efectuados en consumidores de cocaína, en los consumidores jóvenes de marihuana se observa un aumento focal de la función frontal en las áreas 9, 10 y 46 de Brodmann y en el cingulado posterior, que son segmentos del circuito cognitivo, y en el área 23 de Brodmann que es un área de comunicación interhemisférica. También se observan alteraciones funcionales en forma de hipofunción multifocal de distribución desorganizada en fumadores de marihuana, aunque de menor significado estadístico (menor severidad) que en los consumidores de cocaína.

Estos hallazgos nos permiten plantear la presencia de neurotoxicidad en los consumidores de marihuana ya que, al comparar sus resultados con una base de datos normativa para personas del mismo grupo etario, ninguno de los estudios de NeuroSPECT de los jóvenes consumidores fue normal. Cabe agregar que los jóvenes reportaron haberla consumido la misma semana del examen del NeuroSPECT.

Todo esto incide en que las expectativas de cursar estudios superiores universitarios en el grupo de consumidores son significativamente más bajas, con un $21 \%$, comparado con un $43 \%$ para los no consumidores, de acuerdo a lo ya reportado por Dörr et al. ${ }^{3}$

Los resultados de las pruebas de neuroimagen, que muestran efectos en áreas del cerebro relacionadas con el aprendizaje, son altamente coincidentes con los puntajes obtenidos por los mismos sujetos en las pruebas neuropsicológicas, lo que agrega evidencia a los efectos negativos del consumo de marihuana en el aprendizaje, tema central de este estudio. Especialmente importante es el hecho de que estos resultados corresponden a adolescentes que no han sido diagnosticados ni rotulados como adictos y que por lo tanto no constituyen aún un problema de salud pública, ni son percibidos como adolescentes en riesgo social. Sin embargo, estos jóvenes provienen de poblaciones socialmente vulnerables asociadas a la pobreza, lo cual agrava las consecuencias o efectos que puede tener para ellos el fracaso escolar ligado al consumo habitual de marihuana, pensando que la educación debería ser el medio que les permita una mayor movilidad social.

La no percepción de riesgo en el consumo, el fácil acceso a la cannabis, el incuestionable efecto nocivo sobre las funciones cognitivas involucradas en el aprendizaje y el desempeño escolar, el aumento del número de mujeres adolescentes consumidoras, la evidencia reunida sobre la cannabis como inductora o facilitadora del uso de otras sustancias y la disminución de la edad de inicio del consumo, constituyen un problema para las políticas de salud pública, que compromete a la escuela, a los adolescentes y a sus familias. En relación a este desafío, se constata nuevamente la deuda de las instituciones, en especial de la familia y de la escuela, en el sentido de que los adolescentes consumidores no perciben conciencia de riesgo por parte de sus padres o de sus profesores, ni control social sobre el consumo.

\section{AGRADECIMIENTOS}

Los autores agradecen la colaboración de Julio Figueroa y Jair López. 


\section{REFERENCIAS}

1. Informe Mundial Sobre las Drogas, 2010. Naciones Unidas. Oficina contra la Droga y el Delito; http://www.unodc.org/documents/wdr/ WDR_2010/World_Drug_Report_2010_lo-res.pdf, 26 de junio de 2013.

2. SENDA, Servicio Nacional para la Prevención y Rehabilitación del Consumo de Drogas y Alcohol. Noveno estudio nacional de drogas en población escolar. Principales resultados. Septiembre, 2012; http:// www.senda.gob.cl/wp-content/uploads/2012/09/Noveno_Estudio_Escolares_Informe_Ejecutivo.pdf, 26 de junio de 2013.

3. Dörr A, Gorostegui ME, Viani S, Dörr MP. Adolescentes consumidores de marihuana: implicancias para la familia y la escuela. Salud Mental 2009;32(4):269-278.

4. Conace. Octavo estudio nacional de drogas en población escolar de Chile. 2009; http://www.senda.gob.cl/wp-content/uploads/2011/04/2009_ octavo_estudio_escolar.pdf, 26 de junio de 2013.

5. Comisión Clínica de la Delegación de Gobierno para el Plan Nacional sobre Drogas. Informe sobre Cannabis. España, Ministerio de Sanidad y Consumo. Febrero 2006; http://www.pnsd.msc.es/Categoria2/publica/pdf/InformeCannabis.pdf, 26 de julio de 2013.

6. Degenhardt L, Chiu WT, Sampson $\mathrm{N}$ et al. Toward a global view of alcohol, tobacco, cannabis, and cocaine use: findings from the WHO World Menthal Health Surveys. PloS Med 2008;5(7):e141.

7. Breslau J, Miller E, Joanie Chung WJ, Schweitzer JB. Childhood and adolescent onset psychiatric disorders, substance use, and failure to graduate high school on time. J Psychiatr Res 2011;45(39):295-301.

8. Borges G, Mora-Icaza ME, Benjet C, Lee S et al. Influence of mental disordes on school dropout in Mexico. Rev Panam Salud Publica 2011;30(5):477-483.

9. Ferguson DM, Horwood LJ, Beautrais AL. Cannabis and educational achievement. Addiction 2003;98(12):1681-1692.

10. Caspi A. Moffitt TE. Cannon M, McClay J et al. Moderation of the effect of adolescent-onset cannabis use on adult psychosis by a functional polymorphism in the catechol-O-methyltransferase gene: longitudinal evidence of a gene $\mathrm{X}$ environment interaction. Biol Psychiatry 2005;57(10):1117-1127.

11. Lundqvist $T$, Jönsson $S$, Warkentin $S$. Frontal lobe dysfunction in long-term cannabis users. Neurotoxicol Teratol 2001;23:437-443.

12. Volkow ND, Gillespie H, Mullani N, Tancredi L et al. Brain glucose metabolism in chronic marijuana users at baseline and during marijuana intoxication. Psychiatry Res 1996;67:29-38.
13. Block RI, Erwin WJ, Ghoneim MM. Chronic drug use and cognitive impairments. Pharmacol Biochem Behav 2002;73(3):491-504.

14. American Psychiatric Association. DSM-IV-TR Diagnostic and Statistical Manual of Mental Disorders. Cuarta edición -TR. Washington, DC; 2000.

15. Quiroga GM. Cannabis y psicopatología comórbida. Revista Adicciones 2002;14:(2)191-200.

16. Verdejo-García A, Pérez-García M, Sánchez-Barrera M, RodríguezFernández A et al. Neuroimagen y drogodependencias: correlatos neuroanatómicos del consumo de cocaína, opiáceos, cannabis y éxtasis. Rev Neurol 2007;44:432-439.

17. Holman L, Carvalho P, Mendelson J, Teoh SK et al. Brain perfusion is abnormal in Cocaine-Dependent Polydrug users: A study using Technetium-99m-HMPAO and ASPECT. J Nucl Med 1991;32:1206-1210.

18. Miller BL, Mena I, Giombetti R, Villanueva-Meyer J et al. Neuropsychiatric effects of cocaine: SPECT measurements. J Addict Dis 1992;11:47-58.

19. Thomas C, Mena I, Chang L, Strickland T. Effects of cocaine in brain perfusion imaging. Clin Nucl Med 1995;20(9):855.

20. Mena I, Correa R, Nader A. Alteraciones neuro-funcionales en trastornos del ánimo que cursan con conductas auto-mutilatorias: estudio de perfusión regional cerebral a partir de la técnica de Neuro-SPECT Tc99HMPAO. Alasbimn J 9(36): April 2007. Article N॰ AJ36-2; http://www2. alasbimnjournal.cl/alasbimn/CDA/sec_a/0,1205,SCID\%253D19959\%25 26PRT\%253D19905\%2526LNID\%253D33,00.html, 22 de julio de 2013.

21. Wilson W, Mathew R, Turkington T, Hawk T et al. Brain morphological changes and early marijuana use: a magnetic resonance and positron emission tomography study. J Addict Dis 2000;19:1-22.

22. Finger B, Knoch D, Johnson EJ, Krosch AR et al. Lateral prefrontal cortex and self-control in intertemporal choice. Nature Neuroscience 2010;13:538.

23. Kable JK. Just a little (lateral prefrontal) Patience. Nature Neuroscience 2010;13:523.

24. Strauch B. The primal teen. What the new discoveries about the teenage brain tell us about our kids. Nueva York: Anchor Books; 2003.

25. Ranganathan M, D'Souza DC. The acute effects of cannabinoids on memory in humans: a review. Psychopharmacology (Berl) 2006;188(4):425-444.

26. Block RI, Ghoneim MM. Effects of chronic marijuana use on human cognition. Psychofarmacology (Berl) 1993;110(1-2):219-228.

27. Adimark. Mapa socieconómico de Chile, 2002; http://www.adimark.cl/ medios/estudios/Mapa_socieconómico_de_Chile.pdf, 6 de junio de 2011.

Artículo sin conflicto de intereses 